\title{
Caracterización y delimitación del trastorno de la comunicación social (pragmático)
}

\section{Characterization and definition of social communication disorder (pragmatic)}

\author{
Mónica González Blanco, Rosa Mª Rivas Torres y Santiago López Gómez \\ Dpto. Psicología Evolutiva y de la Educación, Universidad de Santiago de Compostela
}

\begin{abstract}
Resumen
Los déficits en la comunicación social son tipificados por primera vez en las clasificaciones internacionales en el DSM-5, bajo el epígrafe de Trastorno de comunicación social (pragmático) (TCS). El objetivo de esta investigación es delimitar los criterios bajo los cuales cabe establecer el diagnóstico de los sujetos que presentan signos de disfuncionalidad pragmática, considerando los distintos contextos, sociales, situacionales o comunicativos en los que se pueden dar. La problemática observada en las diversas clasificaciones está en sus límites difusos, encuadrándose como sintomatología de otros trastornos, principalmente dentro de TEA y del TEL. En la actualidad el TCS se caracteriza por déficits con la pragmática. Dichos déficits se expresan a modo de limitaciones funcionales en la eficacia comunicativa, la participación social, el desarrollo de relaciones sociales, logros académicos o resultados laborales.
\end{abstract}

Palabras clave: Trastorno de la comunicación social. Déficit pragmático. Criterios diagnósticos.

\section{Introducción}

La pragmática y sus alteraciones surgen como campo de estudio del lenguaje, en un primer momento, desde la logopedia, bajo la etiqueta de trastorno semánticopragmático (TSP). Bajo esta etiqueta se ha descrito a un conjunto de alteraciones relacionadas con los usos sociales del lenguaje y la comunicación. Con el tiempo acabaría por convertirse en una categoría diagnóstica independiente del trastorno específico da lenguaje (TEL) y de los trastornos del espectro autista.

Las investigaciones realizadas fueron escasas $y$ mayoritariamente se han basado en descripciones clínicas. Este hecho contribuyó a que se dibujaran múltiples imprecisiones que resalta la falta de una definición y catalogación claras del trastorno. Asimismo, emergieron varios planteamientos que hicieron plantearse diversas cuestiones, sobre todo: ¿El TSP se enmarca dentro del TEL o responde a una problemática del campo cognitivo? ¿Merece una categoría diagnóstica propia?

\section{Antecedentes y evolución del concepto}

$\mathrm{Si}$ bien el TSP tiene una corta trayectoria en el mundo de la clínica del lenguaje (véase Tabla 1), podemos establecer una subdivisión en las grandes ideas que han ido delimitado el trastorno y que se corresponden en un primer momento con las aportaciones de la década de los 80 y 90 , hasta las conceptualizaciones de los primeros años del S. XXI y la gestación del DSM-5.

\section{Conceptualización del TCS en los años 80-90}

Las primeras descripciones del TCS surgen en los primeros años de la década de los años 80. Destacan autores como Rapin y Allen, quienes a partir de diversas observaciones clínicas ofrecen la denominación de síndrome y lo desvinculan del autismo. El síndrome semánticopragmático, enmarcado dentro de los trastornos del desarrollo, se caracteriza por (Rapin y Allen, 1983):

- Fluidez expresiva, pero lenguaje poco comunicativo.

- Adecuada morfosintaxis y fonología.

- Déficits en la interpretación y extracción del significado relevante de las conversaciones, así como en la integración en los discursos comunicativos.

- Adecuada comprensión de frases cortas y palabras aisladas, pero dificultades para la comprensión del discurso completo.

- Respuestas no ajustadas o desvinculadas de las preguntas formuladas.

- Cambios repentinos en los temas de conversación.

- Ecolalia y repeticiones.

Años más tarde Bishop y Rosenbloom (1987) rechazan esta etiqueta retomando la denominación de trastorno. El mismo vendría definido por dificultades de comprensión y producción (concretamente: respuestas no ajustadas a las preguntas formuladas, uso de expresiones irrelevantes, dificultades para interpretar la información conversacional relevante). Sus rasgos clínicos, serían:

- Retraso en el desarrollo del lenguaje.

- Detección de más errores de comprensión que de expresión.

- Atracción por los sonidos sin tener en cuenta su significado.

- Alteraciones en las percepciones de objetos o situaciones.

- Dificultades para encontrar la palabra idónea en un contexto concreto.

- Planteamiento de preguntas de las cuales no se esperan ni se desea respuesta.

- Problemas para producir y comprender gestos, expresiones faciales, o la prosodia.

- Fluidez expresiva pero déficits en sintaxis y fonología.

- Comprensión literal no detectando la ironía, el sarcasmo y/o las metáforas.

- No se manifiesta o se da escasamente o juego imaginativo

- Problemas de comprensión lectora.

- Falta de atención.

- Problemas de conducta.

- Posibles problemas de motricidad gruesa.

- Dificultades para comprender una conversación o un hecho cuando se enmarca o hace referencia a situaciones espontáneas y no estructuradas. 
Esta caracterización de TSP surge de descripciones clínicas y se basa en hipótesis diagnósticas. Pero carecía de unos criterios objetivos y tampoco existía una evaluación estandarizada y específica del mismo. Por todo ello, el TSP tenía unos límites poco precisos siendo complicado, en ciertos casos, establecer la diferencia con la normalidad o evitar la confusión con otros trastornos como o síndrome de LandauKleffner o el síndrome de Williams.

Bishop y Adams (1989) diseñaron un procedimiento semiestructurado de evaluación de habilidades pragmáticas basado en la grabación, transcripción y análisis de conversaciones niño-adulto. Los niños con TSP se describen como aquellos que interrumpen a menudo, presentan gran cantidad de inicios conversacionales, inadecuaciones conversacionales y dificultades para la comprensión del significado no literal. Las reacciones adultas implican múltiples aclaraciones del contenido comunicativo, dada su poca compresión.

No obstante, y pese a los notables esfuerzos descriptivos, queda en evidencia la falta de aclaración de la problemática que se manifiesta en estos niños. Tampoco se aclara si es debida a problemas lingüísticos o a déficits cognitivos $\mathrm{y}$, tal vez lo más importante, si el trastorno forma parte del autismo.

En la década dos 90 las investigaciones llevadas a cabo relacionan el TSP desde la "teoría da mente", concretamente entre la teoría de la mente y el lenguaje como habilidades vinculadas entre sí. A partir de tareas mentalistas y usando pruebas de creencias falsas se detectó que los niños con TSP tenían dificultades en tareas de creencias falsas de segundo orden, que se relacionan directamente con una falta de atribución de creencias falsas en los demás (Sullivan, Zaitchik \& Tager-Plusberg, 1994), siendo comparables estas problemáticas con las dificultades que presentan los niños autistas o con trastornos que limitan con el espectro autista, como el síndrome de Williams (Sullivan \& Tager-Plusberg, 1999). Este hecho hizo que el TSP pudiese considerarse como un trastorno intermedio entre o TEL y el autismo, recogiendo síntomas de ambos, pero sin cumplir con todos sus criterios diagnósticos específicos.

\section{La conceptualización del TCS en el S. XXI}

Bishop (2000), ya en el S. XXI, fija una etiqueta más precisa y concreta, introduciendo la etiqueta de trastorno pragmático del lenguaje (TPL). El TPL incluye tan solo dificultades de tipo pragmático. Separa los ámbitos pragmático y semántico, puesto que en sus estudios se detectaron casos de individuos que presentaban alteraciones de tipo comunicativo, pero con plenas competencias a nivel semántico. En el 2004, establece una nueva subdivisión en el trastorno específico del lenguaje (TEL), diferenciando 4 subgrupos:

- TEL con problemas de producción.

- TEL predominantemente gramatical.

- TEL con graves problemas de comprensión del lenguaje.

- TEL con problemas fundamentalmente pragmáticos.

Botting \& Conti-Ramsdem (2003) establecen una división dentro del trastorno pragmático del lenguaje (TPL), diferenciando dos subgrupos que difieren en características y dimensiones etiológicas: por un lado el TPL-plus: próximo al perfil del TEA (con marcados déficits comunicativos); por otro, el TPL-pur: próximo al perfil TEL (con marcadas dificultades en el procesamiento semántico y pragmático en contextos y situaciones complejas).

Por su parte Mulas, Etcheporeborda, Díaz-Lucero y Ruíz-Andrés (2006) ofrecen una nueva visión del déficit semántico-pragmático caracterizándolo como una alteración que conlleva la interpretación literal de los mensajes verbales y la no respuesta idónea a las preguntas que le son formuladas al sujeto puesto que este no tiene en cuenta a mensaje completo, tan solo tiene presente uno o varios conceptos que componen el mismo. El habla no se ve alterada y es fluida y correcta, aunque pueden registrarse ecolalias y perseveraciones.

Tabla 1. Evolución de la conceptualización del trastorno de la comunicación social

\begin{tabular}{|c|c|c|}
\hline $\begin{array}{l}\text { Rapin \& } \\
\text { Allen (1983) }\end{array}$ & $\begin{array}{l}\text { Definición del trastorno } \\
\text { específico del Lenguaje } \\
\text { (TEL) }\end{array}$ & $\begin{array}{l}\text { Dificultades gramaticales y } \\
\text { pragmáticas para adquirir la } \\
\text { primera lengua descartando } \\
\text { alteraciones orgánicas o retraso } \\
\text { mental. } \\
\text { Incluyen el síndrome } \\
\text { semántico-pragmático }\end{array}$ \\
\hline $\begin{array}{l}\text { Bishop e } \\
\text { Rosenbloom } \\
\text { (1987) }\end{array}$ & $\begin{array}{l}\text { Trastorno semántico- } \\
\text { pragmático del lenguaje } \\
\text { (TSP) }\end{array}$ & $\begin{array}{l}\text { Dificultades comunicativas de } \\
\text { índole semántico e pragmático }\end{array}$ \\
\hline $\begin{array}{l}\text { Bishop } \\
(2000)\end{array}$ & $\begin{array}{l}\text { Trastorno pragmático } \\
\text { da lenguaje (TPL) }\end{array}$ & $\begin{array}{l}\text { Dificultades comunicativas } \\
\text { pero competencia semántica. } \\
\text { Empobrecimiento de la } \\
\text { representación simbólica de las } \\
\text { emociones. }\end{array}$ \\
\hline $\begin{array}{l}\text { Botting \& } \\
\text { Conti- } \\
\text { Ramsdem } \\
(2003)\end{array}$ & $\begin{array}{l}\text { TPL-plus } \\
\text { TPL-pur }\end{array}$ & \\
\hline $\begin{array}{l}\text { Bishop } \\
\text { (2004) }\end{array}$ & $\begin{array}{l}\text { TEL con problemas de } \\
\text { producción } \\
\text { TEL } \\
\text { predominantemente } \\
\text { gramatical } \\
\text { TEL con graves } \\
\text { problemas } \\
\text { comprensión de } \\
\text { lenguaje del } \\
\text { TEL con problemas } \\
\text { fundamentalmente } \\
\text { pragmáticos }\end{array}$ & \\
\hline $\begin{array}{l}\text { Mulas et al. } \\
\text { (2006) }\end{array}$ & $\begin{array}{ll}\text { Déficit } & \text { semántico- } \\
\text { pragmático } & \end{array}$ & 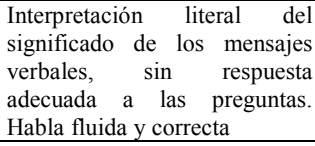 \\
\hline $\begin{array}{l}\text { DSM-5 } \\
(\text { APA, 2013) }\end{array}$ & $\begin{array}{lr}\text { Trastorno de } & \text { la } \\
\begin{array}{l}\text { comunicación } \\
\text { (pragmático) }\end{array} & \text { social }\end{array}$ & \\
\hline
\end{tabular}

\section{Conceptualización del TCS}

El trastorno de comunicación social lo presenta un número significativo de sujetos, por lo que conocer sus características, la forma en la que se manifiesta y las consecuencias que puede tener emparejadas es realmente importante.

En la actualidad no existen datos sobre la incidencia concreta del TCS. Castro-Rebolledo, Giraldo-Prieto, Hincapié-Henao, Lopera y Pined (2004) cifran en un 7,4\% la prevalencia del trastorno específico del desarrollo del lenguaje para la población general de niños de 5 anos.

Gassió-Subirachs (2006) indican la prevalencia del trastorno del lenguaje en edad escolar entre un $2 \%$ y un $3 \%$, y el trastorno del habla se situaría entre el 3\% al 6\%. Durante los períodos escolares la prevalencia de ambos trastornos es mayor, rondando conjuntamente el $15 \%$. Con un nivel de incidencia que es superior en varones $(3: 1)$.

El trastorno de la comunicación social (pragmático) (315.39), según el DSM-5 (APA, 2013), se describe bajo el epígrafe de los trastornos de la comunicación y estos, a su vez, forman parte de los trastornos del desarrollo neurológico. El neurodesarrollo se describe como un proceso dinámico, que comienza en la infancia y mediante el cual el cerebro se va desenvolviendo debido al resultado de los numerosos cambios y conexiones derivados de la estimulación ambiental.

Los trastornos del desarrollo neurológico se caracterizan por las alteraciones o retrasos en la maduración do sistema nervioso central debidos a disfunciones 
neurológicas, psíquicas o cognitivas. Las repercusiones de estas problemáticas serán más o menos marcadas segundo el entorno que rodee al sujeto y a la capacidad de adaptación al mismo. Estos déficits pueden afectar a la capacidad del individuo para procesar, interpretar, almacenar o dar respuesta a la información que recibe. Por lo tanto, puede resultar alterada la capacidad intelectual, la comunicación o el aprendizaje.

Los trastornos de la comunicación, dentro del cual se encuentra el TCS, se caracterizan por tener un comienzo temprano. Esto dificulta su detección pues las primeras manifestaciones de déficits pueden ser confundidas bajo la consideración de un posible retraso madurativo o enlentecimiento en la adquisición de habilidades comunicativas. Estas dificultades pueden revertir en alteraciones funcionales presentes a lo largo de la vida, viéndose afectadas tanto conductas verbales como no verbales que repercuten en el comportamiento, las ideas o las actitudes cara a los demás.

\section{El TCS en el DSM-5}

En las diversas apariciones del Manual diagnóstico y estadístico de los trastornos mentales (DSM) no se estableció ninguna etiqueta diagnóstica específica que describiera a aquellos individuos que presentaban dificultades a nivel pragmático.

En el DSM-5 (2013) se establecen cambios a destacar. Los trastornos de la comunicación pasan a presentarse en tan solo tres categorías en función de la presencia de alteraciones:

- Alteraciones en el lenguaje (implican la forma, la función y el uso del lenguaje): recoge al Trastorno del lenguaje, que incluye a las anteriores categorías diagnósticas de trastorno del lenguaje expresivo y el trastorno mixto del lenguaje receptivo-expresivo).

- Alteraciones en el habla (implica a la articulación, la fluidez, la voz y la resonancia): recoge las categorías diagnósticas de trastorno fonológico y tartamudeo.

- Alteraciones en la comunicación (implica a las conductas verbales y no verbales de las interacciones): recoge al Trastorno de la comunicación social (315.39), con dificultades, presentes ya desde edades tempranas, en los aspectos pragmáticos de la comunicación social tanto a nivel expresivo como comprensivo. Pueden o no acompañarse de déficits fonológicos, sintácticos y/o semánticos.

No será pues hasta fechas recientes cuando, después de atribuírsele variadas y diversas etiquetas a lo largo del tiempo, tal como refleja la Tabla 1, el TCS obtenga la consideración de categoría gnoseológica específica. Es necesario conocer con detalle el proceso de adquisición normal de las competencias pragmáticas, para detectar la existencia de déficits en la pragmática y determinar si se cumplen los criterios diagnósticos que el DSM-5 establece.

El TCS está delimitado en el DSM-5 (APA, 2013) por diferentes criterios diagnósticos que lo definen como un conjunto de dificultades persistentes que inciden negativamente en la adquisición y en el uso adecuado del lenguaje, ya sea a nivel oral, escrito o por cualquier otra modalidad de expresión lingüística. Las dificultades vienen dadas por déficits en la comprensión y/o en la producción de diversos elementos del lenguaje, tales como vocabulario reducido, limitada capacidad para emplear palabras y terminaciones para formar oraciones basadas en las reglas gramaticales y morfológicas, así como deficiencias en el discurso (problemas en el uso del vocabulario y de oraciones para explicar o describir un tema o mantener una conversación).

Por tanto, el trastorno de comunicación social viene determinado por una dificultad primaria con la pragmática y la comunicación, provocando diferentes alteraciones: en comprensión, con la presencia de déficits en el seguimiento de las normas sociales, déficits en la comunicación verbal y no verbal en contextos cotidianos. Además, se pueden registrar carencias en la adaptación del lenguaje a las necesidades del oyente o a la situación, así como problemas en el cumplimento de las reglas de comunicación y del discurso.

De manera específica, y siguiendo el DSM-5 (APA, 2013), sus manifestaciones se describen en torno a cuatro grandes criterios diagnósticos, y son:

1. Deficiencias en el uso de la comunicación para propósitos sociales, como saludar y compartir información, de manera que sea apropiada al contexto social.

2. Deterioro de la capacidad para cambiar la comunicación de forma que se adapte al contexto o a las necesidades del que escucha, como hablar de forma diferente en un aula o en un parque, conversar de forma diferente con un niño o con un adulto, y evitar el uso de un lenguaje demasiado formal.

3. Dificultades para seguir las normas de conversación y narración, como respetar el turno en la conversación, expresarse de otro modo cuando no se es bien comprendido y saber cuándo utilizar signos verbales y no verbales para regular la interacción.

4. Dificultades para comprender lo que no se dice explícitamente (p. ej., hacer inferencias) y significados no literales o ambiguos del lenguaje (p. ej., expresiones idiomáticas, humor, metáforas, múltiples significados que dependen del contexto para la interpretación).

Las consecuencias que los citados déficits traen aparejadas causan limitaciones funcionales en la comunicación eficaz, la participación social, las relaciones sociales, los logros académicos o el desempeño laboral, ya sea individualmente o de manera combinada.

Las habilidades del lenguaje van a estar por debajo de lo esperado para la edad cronológica del sujeto, por lo que se registrarán limitaciones funcionales en la comunicación eficaz, en la participación social, en los logros académicos o laborales, tanto a nivel individual como colectivamente. Los síntomas del TCS se manifiestan en edades tempranas, no siendo siempre fáciles de detectar al estar en pleno proceso de desenvolvimiento y adquisición de las habilidades lingüísticas. Los síntomas no se pueden atribuir a la existencia de cualquier tipo de alteración o déficit sensorial, motor o neurológico, así como la presencia de alguna enfermedad o discapacidad intelectual.

\section{Referencias}

American Psychiatric Association (APA). (2013). Diagnostic and statistical manual of mental disorders (5th ed.) (DSM-5). Arlington, VA: American Psychiatric Publishing (Trad. Cast. (2014). Manual Diagnóstico y Estadístico de los Trastornos Mentales (5 ${ }^{a}$ Ed) (DSM-5). Madrid: Editorial Médica Panamericana).

Bishop, D.V.M. (2000). Pragmatic language impairment: a correlate of SLI, a distinct subgroup, or part of the autistic continuum?. En D.V.M. Bishop \& L.B.Leonard (Edit.), Speech and language impairments in children: causes, characteristics, intervention and outcome (pp. 99-113). New York: Psychology Press. 
Bishop, D.V.M. \& Adams C. (1989). Conversational characteristics of children with semantic-pragmatic disorder. 2: What features lead to a judgement of inappropriacy? The British Journal of Disorders of Communication, 24(3), 241-263.

Bishop, D.V.M. \& Rosenbloom, L. (1987). Classification of childhood language disorders. En W. Yule \& M. Rutter (Eds.), Language Development and Disorders. Clinics in Developmental Medicine, 101/102. London: Mac Keith Press.

Bishop, D.V.M. (2004). Specific language impairment: Diagnostic dilemmas. En L. Verhoeven \& H.V. Balkom (Eds.), Classification of developmental language disorders. Theoretical issues and clinical implications (pp. 30-326). New Jersey: Taylor \& Francis.

Botting, N.F. \& Conti-Ramsden, G.M. (2003). Autism, primary pragmatic difficulties, and specific language impairment: can we distinguish them using psycholinguistic markers? Developmental Medicine \& Child Neurology, 45(8), 515-24. Doi: 10.1111/j.14698749.2003.tb00951.x
Castro-Rebolledo, R., Giraldo-Prieto, M., Hincapié-Henao, L., Lopera, F. y Pineda, D.A. (2004). Trastorno específico del desarrollo del lenguaje: una aproximación teórica a su diagnóstico, etiología y manifestaciones clínicas. Revista de Neurología, 39(12), 1173-1181.

Gassió-Subirachs, R. (2006). Trastornos del lenguaje. Anales de Pediatría Continuada, 4(2), 140-144.

Mulas, F., Etcheporeborda, M. C., Díaz-Lucero, A. y RuízAndrés. R. (2006). El lenguaje y los trastornos del neurodesarrollo. Revisión de características clínicas. Revista de Neurología, 42(supl. 2), 103-109.

Sullivan, K., \& Tager-Plusberg, H. (1999). Second-Order Belief Attribution in Williams Syndrome: Intact or Impaired? American Journal of Mental Retardation, 104(6), 523-532. doi: $10.1352 / 0895$ 8017(1999)104<0523:SBAIWS>2.0.CO;2

Sullivan, K., Zaitchik, D. \& Tager-Plusberg, H. (1994). Preschoolers can attribute second-order beliefs. Developmental Psychology, 30, 395-402 